\title{
A Johnson-Kist type representation for truncated vector lattices
}

\author{
Karim Boulabiar* and Rawaa Hajji \\ Laboratoire de Recherche LATAO \\ Département de Mathematiques, Faculté des Sciences de Tunis \\ Université de Tunis El Manar, 2092, El Manar, Tunisia
}

\begin{abstract}
We introduce the notion of (maximal) multi-truncations on a vector lattice as a generalization of the notion of truncations, an object of recent origin. We obtain a Johnson-Kist type representation of vector lattices with maximal multi-truncations as vector lattices of almostfinite extended-real continuous functions. The spectrum that allow such a representation is a particular set of prime ideals equipped with the hull-kernel topology. Various representations from the existing literature will appear as special cases of our general result.
\end{abstract}

Mathematics Subject Classification. 06F20; 46E05; 46A40; 54C30

Keywords. extended-real valued function; Hausdorff; hull-kernel topology; locally compact; multi-truncation; prime (order) ideal; spectrum; truncation; truncated vector lattice; strong truncation; representation; weak truncation.

\section{Introduction}

In his paper 13, Stone investigates the extent to which certains linear functionals on function spaces can be representated as integrals for some measures. It turnd out that the suitable spaces for such representations to hold is

${ }^{*}$ Corresponding author: karim.boulabiar@fst.utm.tn 
the function vector lattices $L$ closed under meat with the constant function 1, i.e., $1 \wedge f \in L$, for all $f \in L$. The classical Daniel-Stone Representation Theorem (see, e.g., Chapter V in [10]) is a remarkable illustration of the importance of these spaces in Measure Theorey. Fremlin, in his book [7], speaks about truncated vector lattices of functions (see also [6]). In the present paper, we shall follow the Fremlin terminology.

We call a truncation on a (real) vector lattice $L$ any (non-zero) function that takes each positive vector $f$ of $L$ to a positive vector $f^{*}$ of $L$, and has the following properties:

(a) If $0 \leq f, g \in L$ then $f \wedge g^{*} \leq f^{*} \leq f$.

$(\mathfrak{b})$ If $0 \leq f \in L$ and $(n f)^{*}=n f$ for all $n \in\{1,2, \ldots\}$, then $f=0$.

By a truncated vector lattice we mean a vector lattice along with a truncation. If in addition the condition

$(\mathfrak{w}) \quad 0 \leq f \in L$ and $f^{*}=0$ imply $f=0$

is fulfilled, we speak about a weak truncation on $L$ and a weakly truncated vector lattice $L$ (this would explain the letter $\mathfrak{w}$ we use to label this third axiom).

In his pioneer works [2, 3], Ball extended the classical Yosida Representation of Archimedean vector lattices with weak units to the wider class of Archimedean weakly truncated vector lattices (Archimedean truncs in Ball's terminology). In this regard, he got a beautiful representation of Archimedean truncs as vector lattices of extended-real valued continuous functions. To be more precise, let $C^{\infty}(\mathcal{X})$ denote the set of all almost-finite extended-real continuous valued functions on a topological space $\mathcal{X}$. Ball proves that for any Archimedean weakly truncated vector lattice $L$, there exists a locally compact Hausdorff space $\mathcal{X}$ such that (i) $L$ is (lattice isomorphic with) a vector lattice of functions in $C^{\infty}(\mathcal{X})$, (ii) $L$ separates points from closed sets in $\mathcal{X}$, and (iii) the truncation on $L$ is provided by meet with the constant function one. A copy of the spectrum $\mathcal{X}$ in Ball's result is a Tychonoff product of spaces of truncation preserving lattice homomorphisms with domain $L$ and codomains totally ordered truncated vector lattices.

Our main purpose in this paper is to extended the Ball Representation Theorem to truncated vector lattices (without assuming the condition $(\mathfrak{w})$ ) 
using a Johnson-Kist type approach (see [9]), which is based on the hullkernel topology on prime ideals rather than the Tychonoff product topology on homomorphisms. Indeed, our central result stipulates that, given an Archimedean truncated vector lattice $L$, a locally compact Hausdorff space $\mathcal{X}$ can be found such that (i) $L$ is identified with a vector lattice of functions in $C^{\infty}(\mathcal{X})$, (ii) $L$ separates points from closed sets in $\mathcal{X}$, (iii) all 'functions' in $L$ vanish at infinity, and (iv) the truncation on $L$ is provided by meet with the characteristic function $1_{\mathcal{Y}}$ of some open-closed set $\mathcal{Y}$ in $\mathcal{X}$. This representation has been made possible through the notion of multi-truncations, which is a 'new' concept involving (maximal) family of truncations on vector lattices. As a matter of fact, a multi-truncation $\mathcal{T}$ on the vector lattice $L$ is just a family of truncations on $L$ with disjoint (i.e., orthogonal) ranges. The idea is to see that the given truncation of the truncated vector lattice $L$ is contained in a maximal multi-truncation $\mathcal{T}$ on $L$. It then turns out that the set

$$
\mathcal{X}=\bigcup_{* \in \mathcal{T}} \bigcup_{f \in L^{+}} \operatorname{Val}\left(f^{*}\right)
$$

along with its hull-kernel topology is a suitable representation spectrum. Here, $\operatorname{Val}\left(f^{*}\right)$ denotes the collection of all prime ideals of $L$ which are maximal with respect to not containing $f^{*}$. As a first application, it will follow that $L$ is a weakly truncated vector lattice if and only if $\mathcal{X}=\mathcal{Y}$, which gives the Ball Representation Theorem referred to above. We shall then focus on the representation of a strongly truncated vector lattice, that is, a truncated vector lattice $L$ for which the extra condition

$(\mathfrak{s})$ if $0 \leq f \in L$ then $(n f)^{*}=n f$ for some $n \in\{1,2, \ldots\}$

is satisfied. Actually, we shall use our previous result (as well as a StoneWeierstrass Approximation Type Theorem) to show that any Archimedean strongly truncated vector lattice $L$ can be embedded as a uniformly dense vector sublattice of the Banach lattice $C_{0}(\mathcal{X})$ of all real-valued continuous functions on $\mathcal{X}$ vanishing at infinity. Moreover, since $L$ is, clearly, a weakly truncated vector lattice, this embedding preserves truncation and its range still separates points from closed sets in $\mathcal{X}$. This latter representation has been obtained in [5] in a completely different way.

Finally, we suggest the reader keeps the textbook [12] by Luxemburg and Zaanen within arm's reach, so it is on hand whenever he needs more information on vector lattices and (order) ideals. 


\section{Tools on truncations and prime ideals}

This section presents the basic properties of truncations which are relevant to our development.

In order to avoid unnecessary repetition, $L$ stands throughout the paper for a vector lattice with $L^{+}$as positive cone.

We start our investigation with the central definition of this paper.

Definition 2.1 By a truncation on $L$ is meant a nonzero function that takes each positive vector $f$ of $L$ to a positive vector $f^{*}$ of $L$ and has the following properties:

(a) $f \wedge g^{*} \leq f^{*} \leq f$ for all $f, g \in L^{+}$.

$(\mathfrak{b})$ If $f \in L^{+}$and $(n f)^{*}=n f$ for all $n \in\{1,2, \ldots\}$ then $f=0$.

It is readily checked that the condition $(\mathfrak{a})$ is met when and only when the condition

$\left(\mathfrak{a}^{\prime}\right) f \wedge g^{*}=f^{*} \wedge g$ for all $f, g \in L^{+}$

is verified. By the way, we shall be free to use, depending on the context, either the condition $(\mathfrak{a})$ or the equivalent version $\left(\mathfrak{a}^{\prime}\right)$.

We record some algebraic identities which will come often in handy.

Lemma 2.2 If $*$ is a truncation on $L$ and $f, g \in L^{+}$then

(i) $(f \wedge g)^{*}=f^{*} \wedge g^{*}$,

(ii) $(f \vee g)^{*}=f^{*} \vee g^{*}$, and

(iii) $\left|f^{*}-g^{*}\right| \leq|f-g|^{*}$.

Proof. (i) It is easily seen that

$$
(f \wedge g)^{*} \leq f^{*} \quad \text { and } \quad(f \wedge g)^{*} \leq g^{*}
$$

Therefore,

$$
(f \wedge g)^{*} \leq f^{*} \wedge g^{*} \leq f \wedge g \wedge g^{*}=(f \wedge g)^{*} \wedge g \leq(f \wedge g)^{*}
$$


which gives the first equality.

(ii) Since $f^{*} \leq f$ and $g^{*} \leq g$, we get

$$
\begin{aligned}
f^{*} \vee g^{*} & =(f \vee g) \wedge\left(f^{*} \vee g^{*}\right)=\left((f \vee g) \wedge f^{*}\right) \vee\left((f \vee g) \wedge g^{*}\right) \\
& =\left((f \vee g)^{*} \wedge f\right) \vee\left((f \vee g)^{*} \wedge g\right)=(f \vee g)^{*} \wedge(f \vee g)=(f \vee g)^{*} .
\end{aligned}
$$

This is the required equality.

(iii) The classical Birkhoff's Inequality (see, e.g., [1, Theorem 1.9]) allows us to write

$$
\begin{aligned}
\left|f^{*}-g^{*}\right| & =\left|f \wedge(f \vee g)^{*}-g \wedge(f \vee g)^{*}\right| \\
& \leq|f-g| \wedge(f \vee g)^{*} \leq|f-g|^{*}
\end{aligned}
$$

This completes the proof of the lemma.

The next lines deal with prime ideals on a vector lattice along with a truncation. Recall here that a vector subspace $P$ of the vector lattice $L$ is called an ideal of $L$ if $P$ contains with any vector $f \in P$ all vectors $g \in L$ such that $|g| \leq|f|$. The ideal $P$ of $L$ is said to be prime if

$$
f, g \in L \text { and } f \wedge g \in P \text { imply } f \in P \text { or } g \in P .
$$

It is not hard to prove that the ideal $P$ is prime if and only if, for every $f, g \in L$ with $f \wedge g=0$, either $f \in P$ or $g \in P$. Also, a necessary and sufficient condition for the ideal $P$ of $L$ to be prime is that $f^{+} \in P$ or $f^{-} \in P$ for any $f \in L$.

The set of all proper prime ideals on $L$ will be denoted by $\mathcal{P}$.

Chapter 5 in [12] contains a thorough study of prime ideals on a vector lattice to which we refer the reader for more information on the subject.

We now list features of prime ideals which will be of great use later.

Lemma 2.3 Let $*$ be a truncation on $L, P \in \mathcal{P}$, and $f, g \in L^{+}$.

(i) If $f-f^{*} \notin P$ then $g^{*}-g \wedge f^{*} \in P$,

(ii) If $\left(f-f^{*}\right)^{*} \wedge\left(g-g^{*}\right)^{*} \notin P$ then $f^{*}-g^{*} \in P$, and

(iii) $f^{*} \in P$ if and only if $(n f)^{*} \in P$ for all $n \in\{1,2, \ldots\}$. 
Proof. (i) Clearly, $f^{*} \wedge g=f^{*} \wedge g^{*}$ and so

$$
g^{*}-g \wedge f^{*}=g^{*}-g^{*} \wedge f^{*}=\left(g^{*}-f^{*}\right)^{+} .
$$

Hence,

$$
\left(g^{*}-g \wedge f^{*}\right) \wedge\left(f-f^{*}\right)=\left(g^{*}-f^{*}\right)^{+} \wedge\left(f-f^{*}\right)=\left(\left(g^{*} \wedge f\right)-f^{*}\right)^{+}=0 .
$$

As $P$ is a prime and $f-f^{*} \notin P$, we get $g^{*}-g \wedge f^{*} \in P$ and (i) follows.

(ii) Applying (i) twice, we see that

$$
f^{*}-f \wedge g^{*} \in P \quad \text { and } \quad g^{*}-g \wedge f^{*} \in P .
$$

As $g \wedge f^{*}=g^{*} \wedge f$, we obtain

$$
f^{*}-g^{*}=\left(f^{*}-f \wedge g^{*}\right)-\left(g^{*}-g \wedge f^{*}\right) \in P,
$$

as desired.

(iii) The 'if' part is obvious. The 'only if' will be established by induction. We have nothing to show for $n=1$. Hence, let $n \geq 2$ and use Lemma 2.2 (iii) to get

$$
0 \leq(n f)^{*}-f^{*} \leq((n-1) f)^{*}
$$

Thus,

$$
0 \leq(n f)^{*} \leq f^{*}+((n-1) f)^{*}
$$

and the proof is complete.

To make the notation less cluttered, if $P \in \mathcal{P}$ and $*$ is a truncation on $L$, we put

$$
\pi^{*}(P)=\left\{u \in L^{+}:\left(u-u^{*}\right)^{*} \notin P\right\} .
$$

Fundamental properties of these sets are given in the last lemma of these preliminaries.

Lemma 2.4 Let $*$ be a truncation on $L$ and $f \in L$.

(i) There exists $n \in\{1,2, \ldots\}$ such that, if $P \in \mathcal{P}$, then

$$
f^{*} \notin P \quad \text { if and only if } n f \in \pi^{*}(P) \text {. }
$$

(ii) If $P \in \mathcal{P}$ and $u, v \in \pi^{*}(P)$, then

$$
\left\{\alpha \in \mathbb{R}:\left(f-\alpha u^{*}\right)^{+} \in P\right\}=\left\{\alpha \in \mathbb{R}:\left(f-\alpha v^{*}\right)^{+} \in P\right\} .
$$


Proof. (i) If $f^{*} \notin P$ then $f>0$. The condition ( $\left.\mathfrak{b}\right)$ ensures the existence of $m \in\{1,2, \ldots\}$ such that $(m f)^{*}<m f$. Put $n=2 m$ and take $P \in \mathcal{P}$. Assume that $n f \in \pi^{*}(P)$, that is, $\left(n f-(n f)^{*}\right)^{*} \notin P$. Since,

$$
0 \leq\left(n f-(n f)^{*}\right)^{*} \leq(n f)^{*}=(n f)^{*} \wedge n f \leq n\left((n f)^{*} \wedge f\right) \leq n f^{*},
$$

we get $f^{*} \notin P$. Conversely, suppose that $f^{*} \notin P$. For brevity, put $g=m f$ and notice that $0<g^{*}<g$. If $g-g^{*} \in P$ then a prime ideal $Q$ of $P$ (and so of $L$ ) can be found so that $g-g^{*} \notin Q$ (see, e.g., [12, Theorem 33.5]). Hence, in any case, there exists a prime ideal $Q$ of $L$ such that

$$
Q \subset P \text { and } g-g^{*} \notin Q
$$

(take $Q=P$ if $g-g^{*} \notin P$ ). If we apply Lemma 2.3 (i) with $Q$ and $2 g$, we obtain

$$
(2 g)^{*}-g^{*}=(2 g)^{*}-\left(g^{*} \wedge 2 g\right) \in Q \subset P .
$$

So, as $g^{*} \notin P$, we get

$$
2 g^{*}-(2 g)^{*}=g^{*}-\left((2 g)^{*}-g^{*}\right) \notin P .
$$

Whence,

$$
\left(2 g^{*}-(2 g)^{*}\right)^{*} \wedge g=\left(2 g^{*}-(2 g)^{*}\right) \wedge g^{*} \notin P,
$$

from which we infer that

$$
\left(2 g^{*}-(2 g)^{*}\right)^{*} \notin P .
$$

On the other hand, from Lemma 2.2 (iii) it follows that

$$
0 \leq(2 g)^{*}=(2 g)^{*}-g^{*}+g^{*} \leq(2 g-g)^{*}+g^{*}=2 g^{*} .
$$

Thus,

$$
0 \leq\left(2 g^{*}-(2 g)^{*}\right)^{*} \leq\left(2 g-(2 g)^{*}\right)^{*}
$$

Accordingly,

$$
\left(n f-(n f)^{*}\right)^{*}=\left(2 g-(2 g)^{*}\right)^{*} \notin P,
$$

and (i) follows.

(ii) Let $u, v \in \pi(P)$ and $\alpha \in \mathbb{R}$. Hence, $u^{*}-v^{*} \in P$ (where we use Lemma 2.3 (ii)), so if $\left(f-\alpha u^{*}\right)^{+} \in P$ then

$$
f-\alpha v^{*}=f-\alpha u^{*}+\alpha\left(u^{*}-v^{*}\right) \leq\left(f-\alpha u^{*}\right)^{+}+\alpha\left(u^{*}-v^{*}\right) \in P .
$$

It follows that $\left(f-\alpha v^{*}\right)^{+} \in P$. Exchanging $u$ and $v$, we can affirm that

$$
\left(f-\alpha u^{*}\right)^{+} \in P \text { if and only if }\left(f-\alpha v^{*}\right)^{+} \in P,
$$

which gives the desired equality. 


\section{Multi-truncations and a spectrum}

Recall that the symbol $\mathcal{P}$ is used to denote the set of all proper prime ideals of the vector lattice $L$. For any non-empty subset $\mathcal{Q}$ of $\mathcal{P}$ and any element $f$ of $L$, we denote by $[\mathcal{Q}]_{f}$ the set of all ideals in $\mathcal{Q}$ which omit $f$, i.e.,

$$
[\mathcal{Q}]_{f}=\{P \in \mathcal{Q}: f \notin P\} .
$$

The family of the sets $[\mathcal{Q}]_{f}$, where $f$ runs through the positive cone $L^{+}$ of $L$, forms a base of the so-called hull-kernel topology on $\mathcal{Q}$. Evidently, the hull-kernel topology on $\mathcal{Q}$ coincides with the topology induced from the hull-kernel topology on $\mathcal{P}$ (for a detailed study of the hull-kernel topology on prime ideals of a vector lattice, the reader is encouraged to consult [12, Section 36]).

From now on, whenever a non-empty subset of $\mathcal{P}$ is considered, it is systematically equipped with its hull-kernel topology as we just explained.

Now we go straight into the second fundamental definition of the paper.

Definition 3.1 We call a multi-truncation on $L$ any family $\mathcal{T}$ of truncations on $L$ such that

$$
f^{*} \wedge f^{\rtimes}=0 \quad \text { for all } f \in L^{+} \text {and } *, \rtimes \in \mathcal{T} \text { with } * \neq \rtimes \text {. }
$$

It is a easy task to check that a family $\mathcal{T}$ of truncations on $L$ is a multitruncation on $L$ if and only if

$$
f^{*} \wedge g^{\rtimes}=0 \quad \text { for all } f, g \in L^{+} \text {and } *, \rtimes \in \mathcal{T} \text { with } * \neq \rtimes \text {. }
$$

This necessary and sufficient condition will be used later without further mention.

The multi-truncation $\mathcal{T}$ is said to be maximal if it is not strictly contained in another multi-truncation on $L$. The following characterizes this special class of multi-truncations in the Archimedean case. Recall first that the vector lattice $L$ is said to be Archimedean if, given $f, g \in L^{+}, n f \leq g$ for all $n \in\{1,2, \ldots\}$, then $f=0$. Obviously, $L$ is Archimedean if and only if

$$
\inf \left\{\frac{1}{n} f: n=1,2, \ldots\right\}=0 \text { for all } f \in L^{+} .
$$


Lemma 3.2 If $L$ is Archimedean then a multi-truncation $\mathcal{T}$ is maximal if and only if, for every $f \in L$ with $f>0$, there exists $* \in \mathcal{T}$ such that $f^{*}>0$.

Proof. Assume that $\mathcal{T}$ is maximal and let $f \in L^{+}$such that $f^{*}=0$ for all $* \in \mathcal{T}$. We must prove that $f=0$. Arguing by contradiction, we suppose that $f>0$. The formula

$$
g^{\rtimes}=f \wedge g \text { for all } g \in L
$$

defines a truncation $\rtimes$ on $L$. Indeed, the condition $(\mathfrak{a})$ being clear, we prove the condition $(\mathfrak{b})$. Let $g \in L^{+}$such that $(n g)^{\rtimes}=n g$ for all $n \in\{1,2, \ldots\}$. Thus, $0 \leq n g \leq f$ for all $n \in\{1,2, \ldots\}$ from which we derive that $g=0$, so $\rtimes$ is a truncation on $L$. Now, if $* \in \mathcal{T}$ and $g \in L^{+}$then

$$
g^{*} \wedge g^{\rtimes}=g^{*} \wedge f \wedge g=f^{*} \wedge g=0 .
$$

It follows that $\mathcal{T} \cup\{\rtimes\}$ is a multi-truncation on $L$. By maximality, we derive that $\rtimes \in \mathcal{T}$ and so $f^{\rtimes}=0$. This yields that $0=f^{\rtimes}=f \wedge f=f$, a contradiction.

Conversely, assume that if $f \in L^{+}$with $f^{*}=0$ for all $* \in \mathcal{T}$, then $f=0$. We claim that $\mathcal{T}$ is maximal. Otherwise, there exists a truncation $\rtimes$ on $L$ such that $\rtimes \notin \mathcal{T}$ and $\mathcal{T} \cup\{\rtimes\}$ is a multi-truncation on $L$. This shows that if $* \in \mathcal{T}$ and $f \in L^{+}$then

$$
0 \leq\left(f^{\rtimes}\right)^{*}=\left(f^{\rtimes}\right)^{*} \wedge f=f^{\rtimes} \wedge f^{*}=0 .
$$

Hence,

$$
\left(f^{\rtimes}\right)^{*}=0 \text { for all } * \in \mathcal{T},
$$

from which it follows that $f^{\rtimes}=0$. We derive that $\rtimes$ vanishes on the whole $L^{+}$, contradicting the definition itself of a truncation.

It should be noted in passing that a standard argument based on Zorn's Lemma yields that any truncation on $L$ is contained in a maximal multitruncation on $L$.

In the rest of the section, we fix a multi-truncation $\mathcal{T}$ on the vector lattice $L$ and a non-empty subset $\mathcal{Q}$ of $\mathcal{P}$.

Define

$$
\mathcal{Q}^{*}=\bigcup_{f \in L^{+}}[\mathcal{Q}]_{f^{*}} \text { for all } * \in \mathcal{T} \quad \text { and } \quad \mathcal{Q}_{\mathcal{T}}=\bigcup_{* \in \mathcal{T}} \mathcal{Q}^{*}
$$

An alternative base of the hull-kernel topology on $\mathcal{Q}_{\mathcal{T}}$ is provided in what follows. 
Lemma 3.3 (i) $\left\{\mathcal{Q}^{*}: * \in \mathcal{T}\right\}$ is a partition of $\mathcal{Q}_{\mathcal{T}}$ into open-closed sets.

(ii) The sets $[\mathcal{Q}]_{f^{*}}$, where $*$ ranges over $\mathcal{T}$ and $f$ ranges over $L^{+}$, form a base for $\mathcal{Q}_{\mathcal{T}}$.

Proof. (i) It only takes a moment's thought to see that for each $P \in \mathcal{Q}_{\mathcal{T}}$ there is exactly one truncation $*$ in $\mathcal{T}$ such that $P \in \mathcal{Q}^{*}$. This means that the family $\left\{\mathcal{Q}^{*}: * \in \mathcal{T}\right\}$ is, indeed, a partition of $\mathcal{Q}_{\mathcal{T}}$. Moreover, if $* \in \mathcal{T}$ and $f \in L^{+}$then

$$
\left[\mathcal{Q}_{\mathcal{T}}\right]_{f^{*}}=\left[\mathcal{Q}^{*}\right]_{f^{*}}=[\mathcal{Q}]_{f^{*}} .
$$

It follows that $[\mathcal{Q}]_{f^{*}}$ is an open set in $\mathcal{Q}_{\mathcal{T}}$ and so is $\mathcal{Q}^{*}$. We infer in particular that the union $\bigcup_{x \in \mathcal{T} \backslash\{*\}} \mathcal{Q}^{\rtimes}$ is again an open set in $\mathcal{Q}_{\mathcal{T}}$. Thus, $\mathcal{Q}^{*}$ is a closed set in $\mathcal{Q}_{\mathcal{T}}$ and (i) follows.

(ii) Choose $f \in L^{+}$and $P \in\left[\mathcal{Q}_{\mathcal{T}}\right]_{f}$. So, there exists $* \in \mathcal{T}$ such that $P \in\left[\mathcal{Q}^{*}\right]_{f}$. In particular, $P \in[\mathcal{Q}]_{g^{*}}$ for some $g \in L^{+}$. Since $P$ is prime, we get

$$
(f \wedge g)^{*}=f^{*} \wedge g^{*}=f \wedge g^{*} \notin P .
$$

Putting $h=f \wedge g$, we derive that

$$
P \in[\mathcal{Q}]_{h^{*}}=\left[\mathcal{Q}^{*}\right]_{h^{*}}=\left[\mathcal{Q}_{\mathcal{T}}\right]_{h^{*}} .
$$

Moreover, if $Q \in\left[\mathcal{Q}_{\mathcal{T}}\right]_{h^{*}}$ then $f \notin Q$ because $h^{*} \leq f$. Consequently, $Q \in$ $\left[\mathcal{Q}_{\mathcal{T}}\right]_{f}$ and thus

$$
P \in\left[\mathcal{Q}_{\mathcal{T}}\right]_{h^{*}} \subset\left[\mathcal{Q}_{\mathcal{T}}\right]_{f} .
$$

This leads to the assertion (ii) and completes the proof of the lemma.

We are going to apply the previous results in a crucial particular case. It is well known that for every $f \in L$ with $f \neq 0$, there exists $P \in \mathcal{P}$ which is maximal with respect to not containing $f$ (for the proof, see [8, Proposition 10.1] or [12, Theorem 33.5 ]). Following the terminology of [4, 8], such an ideal $P$ is referred to as a value of $f$. The (non-empty) set of all values of $f$ is denoted by $\operatorname{Val}(f)$. To proceed our study, we need the following fundamental lemma.

Lemma 3.4 Let $*$ be truncation on $L$ and $f, g \in L^{+}$. Then

$$
\operatorname{Val}\left(f^{*}\right) \cap[\mathcal{P}]_{g^{*}}=\operatorname{Val}\left(g^{*}\right) \cap[\mathcal{P}]_{f^{*}} .
$$


Proof. Let $P \in \operatorname{Val}\left(f^{*}\right)$ and assume that $g^{*} \notin P$. We claim that $P \in$ $\mathrm{Val}\left(g^{*}\right)$. To this end, we shall argue by contradiction supposing that $P \notin$ $\operatorname{Val}\left(g^{*}\right)$. By [12, Theorem 33.5], we can find $Q \in \operatorname{Val}\left(g^{*}\right)$ such that $P \subset Q$. Because $P \neq Q$ and $P \in \operatorname{Val}\left(f^{*}\right)$, we must have $f^{*} \in Q$. Now, from Lemma 2.4 (i) it follows that

$$
\left(m f-(m f)^{*}\right)^{*} \notin P \quad \text { and } \quad\left(n g-\left(n g^{*}\right)\right)^{*} \notin P
$$

hold for some $m, n \in\{1,2, \ldots\}$. Using Lemma 2.3 (ii), we obtain

$$
(m f)^{*}-(n g)^{*} \in P \subset Q .
$$

Furthermore, $(m f)^{*} \in Q$ because $f^{*} \in Q$ (see Lemma 2.3 (iii)). We may conclude that $(n g)^{*} \in Q$. But then $g^{*} \in Q$ which contradicts the condition $Q \in \operatorname{Val}\left(g^{*}\right)$ and shows that $P \in \operatorname{Val}\left(g^{*}\right)$. We infer that

$$
\operatorname{Val}\left(f^{*}\right) \cap[\mathcal{P}]_{g^{*}} \subset \operatorname{Val}\left(g^{*}\right) \cap[\mathcal{P}]_{f^{*}} .
$$

We just swap $f$ and $g$ to get the desired equality.

At this point, we set

$$
\mathcal{V}=\bigcup_{* \in \mathcal{T}} \bigcup_{f \in L^{+}} \operatorname{Val}\left(f^{*}\right)
$$

Observe that from Lemma 3.4 it follows quite easily that

$$
[\mathcal{V}]_{f^{*}}=\operatorname{Val}\left(f^{*}\right) \text { for all } f \in L^{+} \text {. }
$$

Accordingly,

$$
\mathcal{V}^{*}=\bigcup_{f \in L^{+}} \operatorname{Val}\left(f^{*}\right) \text { for all } * \in \mathcal{T} \text { and } \mathcal{V}=\mathcal{V}_{\mathcal{T}}
$$

It turns out that the particular space $\mathcal{V}$, which we call the spectrum of $L$ with respect to the multi-truncation $\mathcal{T}$, enjoys remarkable topological properties.

Lemma $3.5 \quad$ (i) $\mathcal{V}$ is a locally compact Hausdorff space.

(ii) If $L$ is Archimedean and $\mathcal{T}$ is maximal then $\mathcal{V}$ is dense in $\mathcal{P}$. 
Proof. (i) Let $P \in \mathcal{V}$ and $* \in \mathcal{T}$ such that $P \in \mathcal{V}^{*}$. Hence, $P \in \operatorname{Val}\left(f^{*}\right)$ for some $f \in L^{+}$. We call [12, Theorem $\left.36.4(\mathrm{i})\right]$ to affirm that $\operatorname{Val}\left(f^{*}\right)=[\mathcal{V}]_{f^{*}}$ is a compact neighborhood of $P$. This shows that $\mathcal{V}$ is locally compact, as desired. Now, we claim that $\mathcal{V}$ is Hausdorff. To this end, pick $P, Q \in \mathcal{V}$ with $P \neq Q$. Hence, there exist $*, \rtimes \in \mathcal{T}$ and $f, g \in L^{+}$such that

$$
P \in[\mathcal{V}]_{f^{*}}=\operatorname{Val}\left(f^{*}\right) \quad \text { and } \quad Q \in[\mathcal{V}]_{g^{\rtimes}}=\operatorname{Val}\left(g^{\rtimes}\right)
$$

If $* \neq \rtimes$ then the open sets $[\mathcal{V}]_{f^{*}}$ and $[\mathcal{V}]_{g^{\rtimes}}$ are disjoint. Now, suppose that $*=\rtimes$ and let $h=f+g$. Observe that $f^{*} \leq h^{*}$ and so $h^{*} \notin P$. Analogously, we have $h^{*} \notin Q$. In other words, $P, Q \in[\mathcal{V}]_{h^{*}}$ and so $P, Q \in \operatorname{Val}\left(h^{*}\right)$ (where we use Lemma 3.4). Then, by maximality, neither $P \subset Q$ nor $Q \subset P$. Therefore, there exists positive elements $u \in P$ and $v \in Q$ such that $0<v \notin$ $P$ and $0<u \notin Q$. Put

$$
a=u-(u \wedge v) \text { and } b=v-(u \wedge v) .
$$

Observe that $a, b>0$ and $a \wedge b=0$, so $\operatorname{Val}\left(a^{*}\right) \cap \operatorname{Val}\left(b^{*}\right)=\emptyset$ (by primality). Moreover, it is not hard to see that $b^{*} \notin P$ (because $b \notin P$ ) and thus, again by Lemma 3.4, we obtain $P \in \operatorname{Val}\left(b^{*}\right)=[\mathcal{V}]_{b^{*}}$. Similarly, $Q \in \operatorname{Val}\left(a^{*}\right)=[\mathcal{V}]_{a^{*}}$, which shows that $\mathcal{V}$ is Hausdorff, as required.

(ii) Let $f \in L^{+}$such that $f \in P$ for all $P \in \mathcal{V}$. Arguing by contradiction, we assume that $0<f$. As $\mathcal{T}$ is maximal, Lemma 3.2 yields that $0<f^{*}$ for some $* \in \mathcal{T}$. Therefore, we can find $P \in \operatorname{Val}\left(f^{*}\right) \subset \mathcal{V}$. But then $f \notin P$, which is an obvious contradiction. We derive that $\bigcap_{P \in \mathcal{V}} P=\{0\}$ and density follows from [12, Theorem 36.1].

\section{Multi-truncations and extended-real valued functions}

We shall keep the same notations of the previous section. On the other hand, we denote by $\overline{\mathbb{R}}$ the two-point compactification of the real line $\mathbb{R}$.

As in the previous section, $\mathcal{T}$ is a multi-truncation on $L$ and $\mathcal{Q}$ is a non-empty subset $\mathcal{Q}$ of $\mathcal{P}$.

According to the first assertion in Lemma 3.3, any function on $\mathcal{Q}_{\mathcal{T}}$ can be defined by its respective restrictions to the sets $\mathcal{Q}^{*}$, where $*$ runs through 
$\mathcal{T}$. In so doing, for any $f \in L$, we define an extended-real valued function $\widehat{f}: \mathcal{Q}_{\mathcal{T}} \rightarrow \overline{\mathbb{R}}$ as follows. For every $P \in \mathcal{Q}_{\mathcal{T}}$, there exists a unique $* \in \mathcal{T}$ such that $P \in \mathcal{Q}^{*}$. Choose then an arbitrary element $u$ in the set

$$
\pi^{*}(P)=\left\{v \in L^{+}:\left(v-v^{*}\right)^{*} \notin P\right\}
$$

and put

$$
\widehat{f}(P)=\inf \left\{\alpha \in \mathbb{R}:\left(f-\alpha u^{*}\right)^{+} \in P\right\} \text { for all } P \in \mathcal{Q}^{*}
$$

(with the usual agreements $\inf \mathbb{R}=-\infty$ and $\inf \emptyset=\infty$ ). Contrary to what may be thought at first glance, Lemma 2.4 (ii) guarantees us that the value of $\widehat{f}$ at the point $P$ does not depend on the choice of $u$ and depends only on $f$ and $P$.

Some of these functions have noteworthy behaviors.

Lemma 4.1 Let $* \in \mathcal{T}$ and $P \in \mathcal{Q}^{*}$. Then

(i) $\widehat{u^{*}}(P)=1$ for all $u \in \pi^{*}(P)$,

(ii) $\widehat{f}(P)=0$ for all $f \in P$, and

(iii) $\widehat{f^{\rtimes}}(P)=0$ for all $f \in L^{+}$and $\rtimes \in \mathcal{T} \backslash\{*\}$.

Proof. (i) Let $u \in \pi^{*}(P)$ and $\alpha<1$. Since

$$
u^{*} \geq\left(u-u^{*}\right)^{*} \notin P,
$$

we see that $u^{*} \notin P$ and so

$$
\left(u^{*}-\alpha u^{*}\right)^{+}=(1-\alpha) u^{*} \notin P .
$$

Moreover, if $\alpha=1$ then $\left(u^{*}-\alpha u^{*}\right)^{+}=0 \in P$ from which we derive that

$$
\widehat{u^{*}}(P)=\inf \left\{\alpha \in \mathbb{R}:\left(u^{*}-\alpha u^{*}\right)^{+} \in P\right\}=1 .
$$

(ii) On the other hand, if $f \in P$ and $\alpha<0$ then

$$
0 \leq-\alpha u^{*} \leq\left(f-\alpha u^{*}\right)^{+}-f .
$$


Therefore, $\left(f-\alpha u^{*}\right)^{+} \notin P$ because $-\alpha u^{*} \notin P$. Furthermore, if $\alpha=0$ then $\left(f-\alpha u^{*}\right)^{+}=f^{+} \in P$. This yields that

$$
\widehat{f}(P)=\inf \left\{\alpha \in \mathbb{R}:\left(f-\alpha u^{*}\right)^{+} \in P\right\}=0 .
$$

(iii) Let $f \in L^{+}$and $\rtimes \in \mathcal{T} \backslash\{*\}$. Clearly, $g^{*} \notin P$ for some $g \in L^{+}$. Since $\rtimes \neq *$, we get $f^{\rtimes} \wedge g^{*}=0$ and so $f^{\rtimes} \in P$. The assertion (ii) leads to the desired conclusion.

Now, we consider the set

$$
\widehat{L}=\{\widehat{f}: f \in L\}
$$

We say that $\widehat{L}$ separates point from closed sets in $\mathcal{Q}_{\mathcal{T}}$ if for every closed set $\mathcal{F}$ in $\mathcal{Q}_{\mathcal{T}}$ and $P \in \mathcal{Q}_{\mathcal{T}}$ with $P \notin \mathcal{F}$, there exists some $f \in L^{+}$such that $f(P)=1$ and $f(Q)=0$ for all $Q \in \mathcal{F}$ (see, e.g., [2, Page 60]). On the other hand, we denote by $1_{D}$ the characteristic (also called indicator) function on any set $D$.

The following lemma plays an essential role in the proof of the main result of this work.

Lemma 4.2 (i) For every $f \in L$, the function $\widehat{f}$ is continuous on $\mathcal{Q}_{\mathcal{T}}$.

(ii) $\widehat{L}$ separates points form closed sets in $\mathcal{Q}_{\mathcal{T}}$.

(iii) $\widehat{f}^{*}=1_{\mathcal{Q}^{*}} \wedge f$ for all $* \in \mathcal{T}$ and $f \in L^{+}$.

Proof. (i) The following proof is, in part, inspired by the proof of [9, Theorem 5.1]. Let $f \in L$ and $P \in \mathcal{Q}_{\mathcal{T}}$. There exists, by Lemma 3.3 (i), a unique $* \in \mathcal{T}$ such that $P \in \mathcal{Q}^{*}$. Select any $u \in \pi^{*}(P)$, so

$$
\widehat{f}(P)=\inf \left\{\alpha \in \mathbb{R}:\left(f-\alpha u^{*}\right)^{+} \in P\right\} .
$$

Observe that $u^{*} \notin P$ because $\left(u-u^{*}\right)^{*} \notin P$ and $u^{*} \geq\left(u-u^{*}\right)^{*}$. Thus,

$$
\widehat{f}(P)=\sup \left\{\alpha \in \mathbb{R}:\left(f-\alpha u^{*}\right)^{-} \in P\right\} .
$$

Indeed, take $\alpha, \beta \in \mathbb{R}$ such that

$$
\left(f-\alpha u^{*}\right)^{+},\left(f-\beta u^{*}\right)^{-} \in P .
$$


We claim that $\beta \leq \alpha$. Otherwise, we would have $\beta-\alpha>0$ and so

$$
\begin{aligned}
0 & <(\beta-\alpha) u^{*}=\left(f-\alpha u^{*}\right)-\left(f-\beta u^{*}\right) \\
& \leq\left(f-\alpha u^{*}\right)^{+}-\left(f-\beta u^{*}\right)^{-} \in P .
\end{aligned}
$$

This contradicts the fact that $u^{*} \notin P$.

Now, suppose that $\widehat{f}(P)=r \in \mathbb{R}$ and take $\varepsilon \in(0, \infty)$. By the previous part, we have

$$
\left(f-(r-\varepsilon) u^{*}\right)^{+} \notin P \quad \text { and } \quad\left(f-(r+\varepsilon) u^{*}\right)^{-} \notin P .
$$

Put

$$
g=\left(u-u^{*}\right)^{*} \wedge\left(f-(r-\varepsilon) u^{*}\right)^{+} \wedge\left(f-(r+\varepsilon) u^{*}\right)^{-}
$$

and notice that $0<g^{*}=g \notin P$ since $P$ is prime. This means that $P \in[\mathcal{Q}]_{g^{*}}$ from which we infer that $[\mathcal{Q}]_{g^{*}}$ is an open neighborhood of $P$ in $\mathcal{Q}_{\mathcal{T}}$ (see Lemma 3.3 (ii)). Moreover, if $Q \in[\mathcal{Q}]_{g^{*}}$ then $\left(u-u^{*}\right)^{*} \notin Q$, so $u \in \pi^{*}(Q)$. Thus, we can write

$$
\begin{aligned}
\widehat{f}(Q) & =\inf \left\{\alpha \in \mathbb{R}:\left(f-\alpha u^{*}\right)^{+} \in Q\right\} \\
& =\sup \left\{\alpha \in \mathbb{R}:\left(f-\alpha u^{*}\right)^{-} \in Q\right\} .
\end{aligned}
$$

Furthermore,

$$
\left(f-(r-\varepsilon) u^{*}\right)^{+} \notin Q \quad \text { and } \quad\left(f-(r+\varepsilon) u^{*}\right)^{-} \notin Q .
$$

We derive directly that

$$
r-\varepsilon \leq \widehat{f}(Q) \leq r+\varepsilon,
$$

which yields that $\widehat{f}$ is continuous in $P$.

Secondly, assume that $\widehat{f}(P)=\infty$, that is,

$$
\left\{\alpha \in \mathbb{R}:\left(f-\alpha u^{*}\right)^{+} \in P\right\}=\emptyset .
$$

Pick a real number $\lambda$ and define

$$
g=\left(u-u^{*}\right)^{*} \wedge\left(f-\lambda u^{*}\right)^{+} .
$$

Consequently, $g^{*}=g \notin P$ and then $[\mathcal{Q}]_{g^{*}}$ is an open neighborhood of $P$ in $\mathcal{Q}_{\mathcal{T}}$. Given $Q \in[\mathcal{Q}]_{g^{*}}$, we see that $\left(f-\lambda u^{*}\right)^{+} \notin Q$ and $\left(u-u^{*}\right)^{*} \notin Q$. It follows that $\widehat{f}(Q) \geq \lambda$, meaning that $\widehat{f}$ is again continuous in $P$. 
Finally, suppose that $\widehat{f}(P)=-\infty$. In other words,

$$
\left\{\alpha \in \mathbb{R}:\left(f-\alpha u^{*}\right)^{+} \in P\right\}=\mathbb{R}
$$

and, by the first case,

$$
\left\{\alpha \in \mathbb{R}:\left((-f)-\alpha u^{*}\right)^{+} \in P\right\}=\emptyset .
$$

Applying the previous case to $-f$, we deduce that $\widehat{-f}$ is continuous on $P$. But then $\widehat{f}$ is also continuous in $P$ because $-\widehat{-f}=-\widehat{f}$ (see Theorem 44.3 (i) in [12]).

(ii) Let $\mathcal{F}$ be a closed set in $\mathcal{Q}_{\mathcal{T}}$ and $Q \in \mathcal{Q}_{\mathcal{T}}$ with $Q \notin \mathcal{F}$. Using Lemma 3.3 (ii), there exists $* \in \mathcal{T}$ and $f \in L^{+}$

$$
Q \in[\mathcal{Q}]_{f^{*}} \text { and } \mathcal{F} \cap[\mathcal{Q}]_{f^{*}}=\emptyset .
$$

By Lemma 2.4 (i), we can find $n \in\{1,2, \ldots\}$ such that $n f \in \pi^{*}(Q)$. Put $u=n f-(n f)^{*}$ and observe that Lemma 4.1 (i) yields that $\widehat{u^{*}}(Q)=1$. Moreover, if $P \in \mathcal{F}$ then $f^{*} \in P$ and so $u^{*} \in P$ (again by Lemma 2.3 (iii)). Using Lemma 4.1 (ii), we obtain $\widehat{u^{*}}(P)=0$ and the required separation property.

(iii) Let $f \in L^{+}$and $* \in \mathcal{T}$. If $P \in \mathcal{Q}_{T}$ with $P \notin \mathcal{Q}^{*}$ then $f^{*} \in P$ and so

$$
\widehat{f}^{*}(P)=0=\left(1_{\mathcal{Q}^{*}} \wedge f\right)(P) .
$$

(where we use Lemma 4.1 (ii)). Now, if $P \in \mathcal{Q}^{*}$ and $u \in \pi^{*}(P)$ then, by Lemma 4.1 (i), we get $\widehat{u}^{*}(P)=1$. Furthermore, Lemma 2.3 (i) shows that $f^{*}-f \wedge u^{*} \in P$. This together with Lemma 4.1 yields that $f^{*}-\overline{-f \wedge} u^{*}(P)=$ 0 . Taking into account elementary identities in [12, Theorem 44.3], we get

$$
\widehat{f^{*}}(P)-\widehat{f}(P) \wedge 1=f^{*} \widehat{-f \wedge} u^{*}(P)=0 .
$$

Thus,

$$
\widehat{f *}(P)=\widehat{f}(P) \wedge 1=\left(1_{\mathcal{Q}^{*}} \wedge \widehat{f}\right)(P),
$$

which gives (iii) and completes the proof of the lemma.

Following [11 and [12, we denote by $C^{\infty}(X)$ the set of all continuous functions $\varphi$ from a topological space $X$ into $\overline{\mathbb{R}}$ such that the set $\{x \in X:|\varphi(x)| \neq \infty\}$ is dense in $X$. Functions in $C^{\infty}(X)$ are usually called almost-finite extendedreal valued continuous functions on $X$. It is well known that $C^{\infty}(X)$ is 
a lattice with respect to the pointwise ordering and if $\varphi \in C^{\infty}(X)$ then $r \varphi \in C^{\infty}(X)$ for all $r \in \mathbb{R}$. However, $C^{\infty}(X)$ need not be a vector lattice, simply because if $\varphi, \psi \in C^{\infty}(X)$ there may not exist a function $\phi$ in $C^{\infty}(X)$ such that $\phi=\varphi+\psi$. In spite of this pathological behavior, we shall allow ourselves to call a vector sublattice of $C^{\infty}(X)$ any sublattice of $C^{\infty}(X)$ which is closed under pointwise addition and scalar multiplication.

Lemma 4.3 If $L$ is Archimedean and $\mathcal{Q}$ is dense in $\mathcal{P}$, then $\widehat{L}$ is a vector sublattice of $C^{\infty}\left(\mathcal{Q}_{\mathcal{T}}\right)$.

Proof. Taking into consideration Lemma 4.2 (i) and Theorem 44.3 in [12] (see also [11, Page 83]), it suffices to show that the open set

$$
\left\{P \in \mathcal{Q}_{\mathcal{T}}:|\widehat{f}|(P) \neq \infty\right\}
$$

is dense in $\mathcal{Q}_{\mathcal{T}}$. Otherwise, the interior $\Omega$ of the set

$$
\left\{P \in \mathcal{Q}_{\mathcal{T}}:|\widehat{f}|(P)=\infty\right\}
$$

is non-empty. Using Lemma 3.3 (ii), there exist $* \in \mathcal{T}$ and $g \in L$ such that $g^{*}>0$ and

$$
[\mathcal{Q}]_{g^{*}}=\left\{P \in \mathcal{Q}: g^{*} \notin P\right\} \subset \Omega .
$$

Let $P \in[\mathcal{Q}]_{g^{*}}$ and observe that $\widehat{|f|}_{*}(P)=\left|\widehat{f}_{*}\right|(P)=\infty$ (where we use [12, Theorem 44.3 (iii)] for the first equality). Moreover, we know that there exists $p \in\{1,2, \ldots\}$ such that $p g \in \pi^{*}(P)$ (see Lemma 2.4 (i)). Putting $u=p g$, we obtain

$$
\inf \left\{\alpha \in \mathbb{R}:\left(|f|-\alpha u^{*}\right)^{+} \in P\right\}=|\widehat{f}|(P)=\infty .
$$

We quickly derive that

$$
\left(n u^{*}-|f|\right)^{+} \in P \text { for all } n \in\{1,2, \ldots\} .
$$

Consequently,

$$
\left(n u^{*}-|f|\right)^{+} \in \bigcap_{g^{*} \notin P \in \mathcal{Q}} P \text { for all } n \in\{1,2, \ldots\} .
$$


However, $\mathcal{Q}$ is dense in $\mathcal{P}$ and so $\bigcap_{P \in \mathcal{Q}} P=\{0\}$ (see [12, Theorem 36.1]). Therefore, by Corollary 35.4 in [12], we get

$$
\left(u^{*}-\frac{1}{n}|f|\right)^{+} \wedge g^{*}=0 \text { for all } n \in\{1,2, \ldots\} .
$$

As $L$ is Archimedean, we infer that

$$
u^{*} \wedge g^{*}=\sup \left\{\left(u^{*}-\frac{1}{n}|f|\right)^{+}: n \in\{1,2, \ldots\}\right\} \wedge g^{*}=0 .
$$

This leads directly to the contradiction

$$
g^{*}=g^{*} \wedge p g=g^{*} \wedge(p g)^{*}=g^{*} \wedge u^{*}=0 .
$$

Accordingly, the open set $\left\{P \in \mathcal{Q}_{\mathcal{T}}:|\widehat{f}|(P) \neq \infty\right\}$ is dense in $\mathcal{Q}_{\mathcal{T}}$, which proves that $\widehat{f} \in C^{\infty}\left(\mathcal{Q}_{\mathcal{T}}\right)$, as desired.

Recall from the previous section that

$$
\mathcal{V}=\bigcup_{* \in \mathcal{T}} \bigcup_{f \in L^{+}} \operatorname{Val}\left(f^{*}\right)
$$

We arrive to the last lemma of this section.

Lemma 4.4 If $* \in \mathcal{T}$ and $f>0$ in $L$, then $\left\{P \in \mathcal{V}^{*}: \widehat{f}^{*}(P)=1\right\}$ is a compact set in $\mathcal{V}$.

Proof. It's easy to see that the set in question, say $\mathcal{K}$, is closed in $\mathcal{V}$. Now take $P \in \mathcal{K}$ and use Lemma 3.4 to see that

$$
P \in \operatorname{Val}\left(g^{*}\right) \cap[\mathcal{P}]_{f^{*}}=\operatorname{Val}\left(f^{*}\right) \cap[\mathcal{P}]_{g^{*}}
$$

holds for some $g \in L^{+}$. This yields that $\mathcal{K} \subset \operatorname{Val}\left(f^{*}\right)$ which is compact (see [12, Theorem $36.4(\mathrm{i})])$.

\section{The main result and its applications}

We have gathered along the previous sections all the ingredients we need to prove the following general representation theorem. 
Theorem 5.1 Let $L$ be an Archimedean vector lattice, $\mathcal{T}$ be a maximal multi-truncation, and $\mathcal{Q}$ be a dense subset of $\mathcal{P}$. Then the map $\Lambda: L \rightarrow$ $C^{\infty}\left(\mathcal{Q}_{\mathcal{T}}\right)$ defined by

$$
\Lambda(f)=\widehat{f} \quad \text { for all } f \in L
$$

is a one-to-one lattice homomorphism such that

$$
\Lambda\left(f^{*}\right)=1_{\mathcal{Q}^{*}} \wedge \Lambda(f) \quad \text { for all } * \in \mathcal{T} \text { and } f \in L^{+} .
$$

Proof. In view of Lemmas 4.24 4.3, and Theorem 44.3 in [12], it only remains for us to show that $\Lambda$ is one-to-one. Let $f \in L$ such that $\widehat{f}(P)=0$ for all $P \in \mathcal{Q}_{\mathcal{T}}$. We claim that $f=0$. To this end, we can assume that $f \in L^{+}$. Suppose by contradiction that $f>0$. The rest of the proof is somewhat reminiscent of the proof of Lemma 4.3. By Lemma 3.2, there exists $* \in \mathcal{T}$ such that $f^{*}>0$. Using Lemma 2.4 (i), we can choose $n \in\{1,2, \ldots\}$ such that $n f \in \pi^{*}(P)$ for any $P \in[\mathcal{Q}]_{f^{*}}$. Put $u=n f$ and take $P \in[\mathcal{Q}]_{f^{*}}$. Thus,

$$
0=\widehat{f}(P)=\inf \left\{\alpha \in \mathbb{R}:\left(f-\alpha u^{*}\right)^{+} \in P\right\} .
$$

Therefore,

$$
\left(f-\frac{1}{n} u^{*}\right)^{+} \in P \text { for all } n \in\{1,2, \ldots\}
$$

We derive that

$$
\left(f-\frac{1}{n} u^{*}\right)^{+} \in \bigcap_{P \in[\mathcal{Q}]_{f^{*}}} P \text { for all } n \in\{1,2, \ldots\} .
$$

Since $\bigcap_{P \in \mathcal{Q}} P=\{0\}$ (by density of $\mathcal{Q}$ in $\mathcal{P}$ ), we can call [12, Corollary 35.4] to write

$$
\left(f-\frac{1}{n} u^{*}\right)^{+} \wedge f^{*}=0 \text { for all } n \in\{1,2, \ldots\} .
$$

Consequently,

$$
f^{*}=f \wedge f^{*}=\inf \left\{\left(f-\frac{1}{n} u^{*}\right)^{+}: n \in\{1,2, \ldots\}\right\} \wedge f^{*}=0
$$

(remember here that $L$ is Archimedean). This contradiction completes the proof of the theorem. 
The maximal orthogonal set (also called a maximal disjoint system) in Theorem 5.1 in [9] (see also Theorem 44.4 in [12]) gives raise to a maximal multi-truncation in an obvious way. This shows directly that Theorem 5.1 is a direct generalization of the classical Johnson-Kist Representation Theorem. The following corollary is also a consequence of Theorem 5.1 combined with Lemma 3.5 (ii).

Corollary 5.2 Let $L$ be an Archimedean vector lattice and $\mathcal{T}$ be a maximal multi-truncation. Then the map $\Lambda: L \rightarrow C^{\infty}(\mathcal{V})$ defined by

$$
\Lambda(f)=\widehat{f} \text { for all } f \in L
$$

is a one-to-one lattice homomorphism such that

$$
\Lambda\left(f^{*}\right)=1_{\mathcal{V}^{*}} \wedge \Lambda(f) \quad \text { for all } * \in \mathcal{T} \text { and } f \in L^{+} .
$$

We are now about to state and prove the main theorem of this research. For, it could be helpful to label the following definition.

Definition 5.3 A vector lattice along with a truncation (in the sense of Definition 2.1) is called a truncated vector lattice.

We proceed to our main result.

Theorem 5.4 For any Archimedean truncated vector lattice L, there exists a locally compact Hausdorff space $\mathcal{X}$ such that

(i) L is (lattice isomorphic with) a vector sublattice of $C^{\infty}(\mathcal{X})$,

(ii) L separates points from closed sets in $\mathcal{X}$,

(iii) There exists an open-closed set $\mathcal{Y}$ in $\mathcal{X}$ such that

$$
f^{*}=1_{\mathcal{Y}} \wedge f \text { for all } f \in L^{+} .
$$

(iv) $L$ vanishes nowhere on $\mathcal{X}$ (i.e., for every $x \in \mathcal{X}$ there exists $f \in L$ such that $f(x) \neq 0)$.

(v) For all $f \in L$ and $\varepsilon \in(0, \infty)$, the set $\{y \in \mathcal{Y}:|f(y)| \geq \varepsilon\}$ is compact. 
Proof. Let $*$ denote the truncation on $L$. As noticed somewhere before, a standard argument based on the Zorn's Lemma shows that there exists a maximal multi-truncation $\mathcal{T}$ on $L$ containing $*$. Put $\mathcal{X}=\mathcal{V}_{\mathcal{T}}=\mathcal{V}$ and $\mathcal{Y}=\mathcal{V}^{*}$. Hence, Corollary 5.2 gives the assertion (i) and (iii). Furthermore, the assertions (ii) and (iv) follow directly from Lemma 4.2 (ii) and Lemma 4.1. respectively. It remains to establish the assertion (v). Choose $f \in L$ and $\varepsilon \in(0, \infty)$. By Lemma 4.4, the set

$$
\mathcal{K}=\left\{P \in \mathcal{V}^{*}: \widehat{\left(\frac{1}{\varepsilon} f\right)}^{*}(P)=1\right\}
$$

is a compact set. Observe now that

$$
\mathcal{K}=\left\{y \in \mathcal{Y}:\left(1_{\mathcal{Y}} \wedge \frac{1}{\varepsilon}|f|\right)(y)=1\right\}=\{y \in \mathcal{Y}:|f(y)| \geq \varepsilon\} .
$$

This completes the proof of the theorem.

Next, we show how we can apply Theorem 5.4 to get two representation theorems from the existing literature. To do this, we first recall the following definition from the introduction.

Definition 5.5 The truncation $*$ on the $L$ is said to be weak if $f=0$ provided $f \in L^{+}$and $f^{*}=0$. In this situation, we call $L$ a weakly truncated vector lattice.

The main part of the following representation theorem is originally due to Ball (see Theorem 5.3.6 in [2]). Here, we furnish an alternative way to get the result.

Theorem 5.6 If $L$ is an Archimedean weakly truncated vector lattice, then there exists a locally compact Hausdorff space $\mathcal{X}$ such that

(i) $L$ is (lattice isomorphic with) a vector lattice of functions in $C^{\infty}(\mathcal{X})$,

(ii) L separates points from closed sets in $\mathcal{X}$,

(iii) $f^{*}=1 \wedge f$ for all $f \in L^{+}$,

(iv) $L$ vanishes nowhere on $\mathcal{X}$, and 
(v) Any $f \in L$ vanishes at infinity.

Proof. By Theorem 5.4, there exists a locally compact Hausdorff space $\mathcal{X}$ such that the conditions (i), (ii), and (iv) are verified. Also, by (iii) in the same theorem, there is an open-closed set $\mathcal{Y}$ of $\mathcal{X}$ such that $f^{*}=1 \mathcal{Y} \wedge f$ for all $f \in L^{+}$. We claim that $\mathcal{Y}=\mathcal{X}$. Otherwise, there exists $x \in \mathcal{X}$ with $x \notin \mathcal{Y}$. Since $\mathcal{Y}$ is a closed set in $\mathcal{X}$ and $L$ separates points from closed sets in $\mathcal{X}$, there exists $f \in L^{+}$such that $f(x)=1$ and $f(y)=0$ for all $y \in \mathcal{Y}$. But then $f^{*}=1 \mathcal{y} \wedge f=0$, while $f \neq 0$. This contradicts the fact that the truncation on $L$ is weak and leads to (iii). This together with Theorem 5.4 (v) proves the last assertion of the theorem.

We conclude the paper with a representation theorem for strongly truncated vector lattices. Although this theorem is an application of the main result of this paper, we shall use also a Stone-Weierstrass type approximation theorem recently obtained in [5]. First, let's introduce strongly truncated vector lattices.

Definition 5.7 The truncation $*$ on $L$ is said to be strong if for every $f \in L^{+}$ there exists $n \in\{1,2, \ldots\}$ such that $(n f)^{*}=n f$. In this case, we call $L$ a strongly truncated vector lattice.

Recall that if $\mathcal{X}$ is a locally compact Hausdorff space, then $C_{0}(\mathcal{X})$ denotes the Banach lattice of all real-valued continuous functions on $\mathcal{X}$ vanishing at infinity. Hence, if $L$ is a vector sublattice of $C_{0}(\mathcal{X})$ such that $1 \wedge f \in L$ for all $f \in L$, then $L$ is uniformly dense in $C_{0}(\mathcal{X})$ if and only if $L$ vanishes nowhere and separates the points of $\mathcal{X}$ (for the proof, see Lemma 4.1 in [5]).

Theorem 5.8 If $L$ is a strongly truncated vector lattice then there exists a locally compact Hausdorff space $\mathcal{X}$ such that

(i) L is (lattice isomorphic with) a uniformly dense vector sublattice of $C_{0}(\mathcal{X})$

(ii) L separates points from closed sets in $\mathcal{X}$, and

(iii) $f^{*}=1 \wedge f$ for all $f \in L^{+}$.

Proof. First, let's see that $L$ is Archimedean. Pick $f, g \in L^{+}$such that $n f \leq g$ for all $n \in\{1,2, \ldots\}$. Since the truncation is strong, we can find 
$p \in\{1,2, \ldots\}$ such that $(p g)^{*}=p g$. Therefore, if $n \in\{1,2, \ldots\}$ then $0 \leq$ $n f \leq p g=(p g)^{*}$ from which we derive that

$$
(n f)^{*}=n f \text { for all } n \in\{1,2, \ldots\} .
$$

This means that $f=0$ and so $L$ is Archimedean, as desired. Moreover, it is readily checked that any strongly truncated vector lattice is a weakly truncated vector lattice. So, by Theorem 5.6, we can say that $L$ is (lattice isomorphic with) a vector sublattice of $C^{\infty}(\mathcal{X})$ for some locally compact Hausdorff space $\mathcal{X}$ such that (ii) and (iii) hold. Moreover, the fact that the truncation is strong together with the condition (iii) yields that all "functions" in $L$ are bounded and so, using Theorem $5.6(\mathrm{v})$, we derive that $L$ is a vector sublattice of $C_{0}(\mathcal{X})$. Now, observe that that $L$ vanishes nowhere and separates the points of $\mathcal{X}$ (where we use Theorem 5.6 (ii) and (iv)). The aforementioned Stone-Weierstrass Approximation Type Theorem ends the proof.

\section{References}

[1] C. D. Aliprantis and O. Burkinshaw, Positive Operators, SpringerVerlag, Dordrecht, 2006.

[2] R. N. Ball, Truncated abelian lattice-ordered groups I: The pointed (Yosida) representation, Topology Appl., 162 (2014), 43-65.

[3] R. N. Ball, Truncated abelian lattice-ordered groups II: The the pointfree (Madden) representation, Topology. Appl., 162 (2014), 56-86.

[4] A. Bigard, K. Keimel, and S. Wolfenstein, Groupes et Anneaux Réticulés, Lecture Notes Math. 608, Springer Verlag, Berlin-Heidelberg-New York, 1977.

[5] K. Boulabiar and R. Hajji, Representation of strongly truncated Riesz spaces, Indag. Math., To appear.

[6] D. H. Fremlin, Measure Theory. Vol. 4, Torres Fremlin, Colchester, 2006.

[7] D. H. Fremlin, Topological Riesz spaces and Measure Theory, Cambridge Univ. Press, Cambridge, 1974. 
[8] M. Darnel, Theory of Lattice-Ordered Groups, Marcel Dekker, New York, 1995.

[9] D. G. Johnson and J. E. Kist, Prime ideals in vector lattices, Can. J. Math., 14 (1962), 517-528.

[10] H. König, Measure and Integration, Springer-Verlag, Berlin-HeidelbergNew York, 1997.

[11] W. A. J. Luxemburg, Some aspects of the theory of Riesz spaces, Univ. Arkansas, Lecture Notes Math., 4. University of Arkansas, Fayetteville, 1979.

[12] W. A. J. Luxemburg and A. C. Zaanen, Riesz Spaces I, North-Holland, Amsterdam-London, 1971.

[13] M. H. Stone, Notes on integration I, Proc. Nat. Acad. Sci. USA, 34 (1948), 336-342. 